\title{
The Combination of Stromal Vascular Fraction Cells and Platelet-Rich Plasma Reduces Malondialdehyde and Nitric Oxide Levels in Deep Dermal Burn Injury
}

\author{
Fonny Josh iD ${ }^{1,2}$ \\ Tomie Hermawan Soekamto ${ }^{3}$ \\ Januar Rizky Adriani ${ }^{4}$ \\ Billy Jonatan ${ }^{4}$ \\ Hiroshi Mizuno ${ }^{5}$ \\ Muhammad Faruk (iD) 4 \\ 'Division of Plastic and Reconstructive \\ Surgery, Department of Surgery, Faculty \\ of Medicine, Hasanuddin University, \\ Makassar, South Sulawesi, Indonesia; \\ ${ }^{2}$ Department of Plastic and \\ Reconstructive Surgery, Wahidin \\ Sudirohusodo Hospital, Makassar, South \\ Sulawesi, Indonesia; ${ }^{3}$ Department of \\ Plastic and Reconstructive Surgery, Koja \\ Hospital, Jakarta, Indonesia; ${ }^{4}$ Department \\ of Surgery, Faculty of Medicine, \\ Hasanuddin University, Makassar, South \\ Sulawesi, Indonesia; ${ }^{5}$ Department of \\ Plastic and Reconstructive Surgery, \\ Juntendo University, Tokyo, Japan
}

\begin{abstract}
Introduction: Thermal burns release reactive oxygen species, which cause profound systemic and local changes. Stromal vascular fraction cells (SVFs) combined with platelet-rich plasma accelerate burn wound healing. This study investigated the effect of a combination of locally injected SVFs and PRP on malondialdehyde (MDA) and nitric oxide (NO) serum and tissue levels in a deep dermal burn model in Wistar rats.

Methods: Thirty-six adult Wistar rats weighing between 150 and 250 grams were used in this study to establish a deep dermal degree burn wound model. They were randomly divided into 4 groups: locally injected the combination SVFs and PRP, the Vaseline group, the placebo group, and healthy Wistar rats (the normal control group). MDA and NO levels in blood serum and burn wound tissue were measured at 8, 24, and 48 hours. Data were analyzed with one-way ANOVA followed by multiple comparisons tests and regression tests. Results: Local injection of SVFs and PRP in combination affected blood MDA, tissue MDA, blood NO and tissue NO levels, with reductions of $0.257 \mu \mathrm{mol} / \mathrm{L}, 0.427 \mu \mathrm{mol} / \mathrm{L}$, $21.78 \mathrm{nmol} / \mathrm{mg}$, and $23.777 \mathrm{nmol} / \mathrm{mg}$, respectively. Injection of SVFs and PRP in combination reduced tissue MDA levels by 1.282 times, NO blood levels by 2.305 , and NO tissue levels by 2.377 times compared to Vaseline application.

Conclusion: The combination of SVFs and PRP undeniably reduced the MDA and NO levels in blood and tissue compared to those in the Vaseline and placebo groups. The injection of these two preparations in combination inhibited the local and systemic stress oxidative response, as illustrated by the decreased MDA and NO levels in blood serum and tissue.
\end{abstract}

Keywords: stromal vascular fraction cells, platelet rich plasma, malondialdehyde, nitric oxide, burn injury

\section{Introduction}

Burn injury is a burden in the health care system, considering its fatal complications, such as shock, electrolyte imbalances, kidney failure and metabolic disorders. ${ }^{1}$ The initial response to thermal trauma involves protein denaturation and cell membrane destruction, which are followed by a change in cytokine levels through the release of proinflammatory mediators such as tumor necrosis factor alpha (TNF- $\alpha$ ) and interleukin (IL) and secretion of reactive oxygen species (ROS) or reactive nitrogen species (RNS). ${ }^{1-3}$
Correspondence: Fonny Josh

Division of Plastic and Reconstructive Surgery, Department of Surgery, Faculty of Medicine, Hasanuddin University, Jalan Perintis Kemerdekaan KM II, Makassar, 90245 , Indonesia

Fax +624II585984

Email fonnyjosh2003@yahoo.com 
ROS and RNS cause the production of free radicals that are responsible for oxidative damage to cellular macromolecules. Oxidative stress arises from the overproduction of ROS and RNS, which are responsible for the pathophysiological changes that occur locally and far from the site of trauma during the development of burn injury. ${ }^{4,5}$ New evidence suggests that overproduction of ROS and RNS leads to cell membrane and nucleic acid destruction, lipid peroxidation, double-strand DNA breaks, singlestrand DNA breaks, and eventually cell apoptosis. ${ }^{6}$

MDA is a secondary product, a metabolite, and an indicator of lipid peroxidation. MDA is formed when free hydroxyl radicals react with fatty acid components, which cause a chain reaction known as lipid peroxidation. This process breaks down fatty acid chains into various toxic products and causes further damage to cell membranes. $^{7}$ On the other hand, nitric oxide (NO) is a physiological mediator that regulates vascular function and inflammation and acts as a neurotransmitter. However, when the homeostasis of the oxygen-oxidation reaction is not well balanced, NO may be converted into proinflammatory and cytotoxic substances through the formation of RNS products. ${ }^{8,9}$

Stromal Vascular Fraction Cells (SVFs) are heterogeneous cells and have the ability to enhance regeneration. ${ }^{10}$ SVFs have been obtained through fat processing via mechanical or enzymatic processes. SVFs may reduce the level of inflammation, as indicated by lower expression of inflammatory cytokines and higher expression of antiinflammatory cytokines (lower IL-6 and TNF- $\alpha$ expression and higher IL-10 expression and M2 macrophage numbers). ${ }^{11-13}$ Platelet Rich Plasma (PRP) is known to play an important role in burn healing, mainly due to the many growth factors it contains. ${ }^{14-16}$ Platelet-derived endothelial cell growth factor (PDGF) and insulin-like growth factor-I (IGF-I) are examples of growth factors that are present in PRP, inhibit the apoptotic pathway during cell turnover and facilitate different stages of wound healing. Both SVFs and PRP have the ability to accelerate epithelialization, induce angiogenesis, and stimulate fibroblasts. ${ }^{17}$ PDGF accelerates wound healing, increases the levels of fibroblast growth factors (FGFs), epidermal growth factors (EGFs), vascular endothelial growth factors (VEGF) and transforming growth factor (TGF). ${ }^{18}$ EGFs scientifically proven accelerate epithelization, which VEGF is an angiogenesis mediator. TGF is a stimulator of fibroblast proliferation and extracellular matrix deposition. ${ }^{14}$ It is expected that the combination of SVFs and PRP will reduce the production of ROS and RNS, followed by a reduction in oxidative stress and cellular damage. This will eventually affect the time needed for the injury to heal and hopefully reduce the burden of burn injury. It is highly expected that local injection of a combination of SVFs and PRP can control the balance of oxidative stress products, which results in decreased systemic inflammatory reactions, prevents multiple organ failure and, more importantly, reduces the mortality rate of burn injury.

This study is a continuation of the previous studies. ${ }^{10,16,17}$ The results obtained from the previous studies which compared the local injection of SVFs, PRP, the combination of SVFs and PRP, and Vaseline (positive control) found that the combination of SVFs and PRP showed a better epithelialization result (complete closure on day 10) compared to SVFs group, PRP group, or Vaseline group alone. In addition, the formation of collagen was thicker, and the number of fibroblasts were higher in the combination group. ${ }^{17}$ Therefore, we focused on further exploring of the combination of SVFs and PRP in reducing oxidative stress (malondialdehyde and nitric oxide level) in the acute phase of burn injury, in line with the previous studies which assessed burn wound healing.

The aim of this study was to investigate the effect of locally injected SVFs and PRP in combination on malondialdehyde (MDA) and nitric oxide (NO) serum and tissue levels in a Wistar rat deep dermal burn model.

\section{Methods}

This research had a posttest control group design and was performed in animals. The study was conducted after obtaining approval from the animal research ethics committee of Hasanuddin University (Code number: 69/ UN4.6.4.5.31/PP36/2020). The laboratory animals are treated according to National Institutes of Health (NIH) guidelines and this work was carried out in line with the ARRIVE Guidelines for Reporting Animal Research. ${ }^{19}$.

\section{Population and Sample}

The subjects were adult male Wistar rats (Rattus norvegicus), 2-3 months old, weighing approximately 150 to 250 grams, all rats were obtained from Hasanuddin University Animal Laboratory. Total thirty-six animals were used in this study, 9 animals for each group (Federer formula). Animals were divided into 4 groups, as follows: the treatment group (locally injected SVFs and PRP in combination), positive control group (topically applied Vaseline), 
negative control group (locally injected placebo), and normal control group (healthy Wistar rats). Sacrifice was performed at 8,24 , and 48 hours after treatment. Wound tissue and serum were collected to assess MDA and NO levels.

\section{Deep Dermal Burn Modeling}

The hair in the back area of the rats was shaved, and the animals were anesthetized with $2 \%$ isoflurane. The shaved area was disinfected with povidone iodine. A deep dermal burn model was established by contacting the back skin of the rat for 15 seconds with a 51-gram, 1-cm-diameter round-bottom metal bar that was previously incubated in $100^{\circ} \mathrm{C}$ hot water for 5 minutes. ${ }^{20}$ The deep dermal burn model was confirmed by histopathological examination with hematoxylin-eosin staining.

\section{Platelet-Rich Plasma Preparation}

PRP was prepared following the Juntendo University Tokyo PRP protocol. In brief, whole blood was drawn preoperatively via cardiac puncture with a $3 \mathrm{~mL} 25 \mathrm{G}$ needle syringe into blood collection tubes containing ethylenediaminetetraacetic acid (EDTA). Thirty milliliters of pooled whole blood from donor rats was collected and then centrifuged for 10 minutes at $2400 \mathrm{rpm}(450 \mathrm{xg})$. The supernatant plasma and buffy coat were collected and centrifuged at $3600 \mathrm{rpm}(850 \mathrm{xg})$ for 15 minutes. After the second centrifugation, $3 \mathrm{~mL}$ of the infranatant plasma containing the buffy coat was reserved as the PRP, which was then frozen in liquid nitrogen and thawed before use. The PRP was then activated by a $10 \% \mathrm{CaCl}_{2}$ solution (Merck, Germany) for transplantation. ${ }^{21}$

\section{Stromal Vascular Fraction Cell Preparation}

SVFs were prepared from the inguinal fat pad of Wistar rats following the established Juntendo University Tokyo SVF protocol. Fat was collected from the right and left groin via an incision. Then, the fat was washed with phosphate-buffered saline (PBS) solution (Gibco-BRL, Grand Island, NY, USA). Finely minced fat tissue was placed into a $15 \mathrm{~mL}$ tube, and $0.15 \%$ collagenase (Wako, Osaka, Japan) was added. After incubation at $37^{\circ} \mathrm{C}$ for 30 minutes, control medium (Dulbecco's modified Eagle's medium [DMEM, Gibco-BRL] and 1\% antibiotic-antimycotic (Sigma-Aldrich, St Louis, USA) supplemented with
$10 \%$ fetal bovine serum (Gibco-BRL) were added to neutralize the collagenase effect. After being centrifuged for 5 minutes at $1.500 \mathrm{RPM}$, cell pellets were resuspended in sterile water. The number of SVFs was counted with Trypan blue (Gibco-BRL) and Neubauer counting chambers. A total of 50,000 SVF cells were transferred into an Eppendorf tube, and PRP was added until a volume of $500 \mu \mathrm{L}$ was reached. ${ }^{22}$ All SVF procedures were performed at Hasanuddin University Medical Research Center (HUM-RC). ${ }^{16}$

\section{Topical Injection, Vaseline, and Placebo Application Method}

Intradermal injection of a combination of SVFs and PRP (treatment group) was performed at the 12, 3, 6, and 9 o'clock positions, and one injection was performed at the center of the wound. A total of $500 \mu \mathrm{L}$ of SVFs and PRP in combination was injected for each wound, with $100 \mu \mathrm{L}$ for each point. In the positive control group, Vaseline petroleum jelly was topically applied to the wound surface. In the placebo group, injured rats were injected intradermally with $500 \mu \mathrm{L}$ of sterile water using the same approach used for the treatment group.

All wounds were then covered with transparent film, which was then placed in a circular corset covering all the wounds. Antibiotics and analgesics were given to all animals. After 8, 24, and 48 hours, animals in each group were sacrificed.

\section{Malondialdehyde and Nitric Oxide Measurements}

Before sacrifice, all animals were anesthetized with $2 \%$ isoflurane. They underwent thoracotomy, and blood was collected using a $3 \mathrm{~mL} 25 \mathrm{G}$ needle syringe, followed by injection of formalin. Skin burn wound tissues were subjected to MDA and NO tissue level measurements; MDA and NO levels in blood and tissue were measured at various time points (8, 24, and 48 hours). Enzyme-linked immunosorbent assay (ELISA) was used for measurements as described in the rat MDA kit protocol (Bioassay Technology Laboratory) and the nitric oxide ELISA kit protocol (Bioassay Technology Laboratory, Shanghai, China). The MDA level was measured in $\mu \mathrm{mol} / \mathrm{L}$, and the NO level was measured in $\mathrm{nmol} / \mathrm{mg}$. ELISA was performed at Hasanuddin University Medical Research Center (HUM-RC). 


\section{Statistical Analysis}

Data were divided based on type and presented as graphs and tables. The data were subjected to statistical analysis using SPSS version 25 (IBM Corp.). Normality tests were carried out using the Shapiro-Wilk test followed by a homogeneity test. Then, the data were analyzed using oneway ANOVA, and the least significance difference (LSD) was used as a multiple comparisons test. A regression test was conducted to analyze the effect of the combination of SVFs and PRP on MDA and NO levels.

\section{Results}

\section{Burn Modeling}

Skin burn tissue was subjected to histological examination and stained with hematoxylin-eosin (HE). The results showed full destruction of the epidermal and dermal layers of the skin with preservation of the hypodermal layer (Figure 1).

\section{Blood Malondialdehyde Level After 8 Hours}

Based on Table 1, the highest blood MDA level was observed at 8 hours in the negative control group, with an average of $0.813 \mu \mathrm{mol} / \mathrm{L} \pm 0.078$, and the lowest average blood MDA level was in the treatment group, with a value of $0.550 \mu \mathrm{mol} / \mathrm{L} \pm 0.113$.

One-way ANOVA showed that there was a significant difference $(p=0.014)$ between interventions (Table 1). The least significance difference (LSD) test showed that the treatment group was significantly different from the negative control group $(p=0.005)$ but not significantly different from the positive control group or normal control group ( $\mathrm{p}=0.453$ and 0.962 , respectively) (Figure 2 ). The positive control group was significantly different from the negative control group, with $\mathrm{p}=0.014$; nevertheless, the positive control group was not significantly different from the treatment group or the normal control group, with p-values of 0.453 and 0.481 , respectively.

\section{Blood Malondialdehyde Level at 24 Hours}

Based on Table 1, the highest average blood MDA level at 24 hours was in the negative control group $(0.820 \mu \mathrm{mol} / \mathrm{L} \pm$ $0.171)$. The lowest average blood MDA level at 24 hours was in the treatment group $(0.537 \mu \mathrm{mol} / \mathrm{L} \pm 0.185)$. Oneway ANOVA demonstrated that $\mathrm{p}=0.080$, so it can be concluded that there was no significant difference between the groups (Figure 2).

\section{Blood Malondialdehyde Level at 48 Hours}

The highest average blood MDA level at 48 hours was observed in the positive control group $(0.880 \mu \mathrm{mol} / \mathrm{L} \pm$ 0.072), and the lowest average blood MDA level was in the normal control group $(0.550 \mu \mathrm{mol} / \mathrm{L} \pm 0.069)$, followed by the treatment group (Table 1). Based on the results of one-way ANOVA, the $\mathrm{p}$-value was smaller than $\alpha$ (p = 0.004), so it can be concluded that there was a significant difference within interventions. The treatment group differed significantly from the positive control group and negative control group, with significance values of 0.004 and 0.015 , respectively. The treatment group was not significantly different from the normal control group, with a significance value of 0.559 . The positive control group differed significantly from the treatment group and the normal control group, with significance values of 0.004 and 0.002 , respectively. The positive control group was not notably different from the negative control group, with $\mathrm{p}=0.375$ (Figure 2).

\section{Tissue Malondialdehyde Level at 8 Hours} Table 1 shows that the highest average tissue MDA level at 8 hours was in the negative control group $(0.843 \mu \mathrm{mol} / \mathrm{L} \pm$ 0.125), and the lowest average tissue MDA level was in the treatment group $(0.593 \mu \mathrm{mol} / \mathrm{L} \pm 0.050)$. One-way ANOVA showed a value of $p=0.036$, and it can be concluded that there was a significant difference between intervention groups. A further test was carried out using the LSD test with the following results: the treatment group differed significantly from the negative control group, with $\mathrm{p}$-value $=$ 0.007 . The treatment group was not significantly different from the positive control group and normal control group, with significance values of 0.217 and 0.389 , respectively. The positive control group was not significantly different from the negative control group, with a significance value of 0.054 , and the positive control group was not significantly different from the treatment group $(p=0.217)$ or normal control group $(p=0.678)$. The negative control group was significantly different from normal control group $(\mathrm{p}=0.028)$ (Figure 3$)$.

\section{Tissue Malondialdehyde Level at 24 Hours}

Based on Table 1, the highest average 24-hour tissue MDA level was in the negative control group $(0.767 \mu \mathrm{mol} / \mathrm{L} \pm$ 0.081 ), and the lowest was in the normal control group $(0.650 \mu \mathrm{mol} / \mathrm{L} \pm 0.096)$. The data were normally distributed and homogeneous ( $>0.05)$. Based on the results of one-way ANOVA, the p-value was greater than $\alpha(0.339>$ 


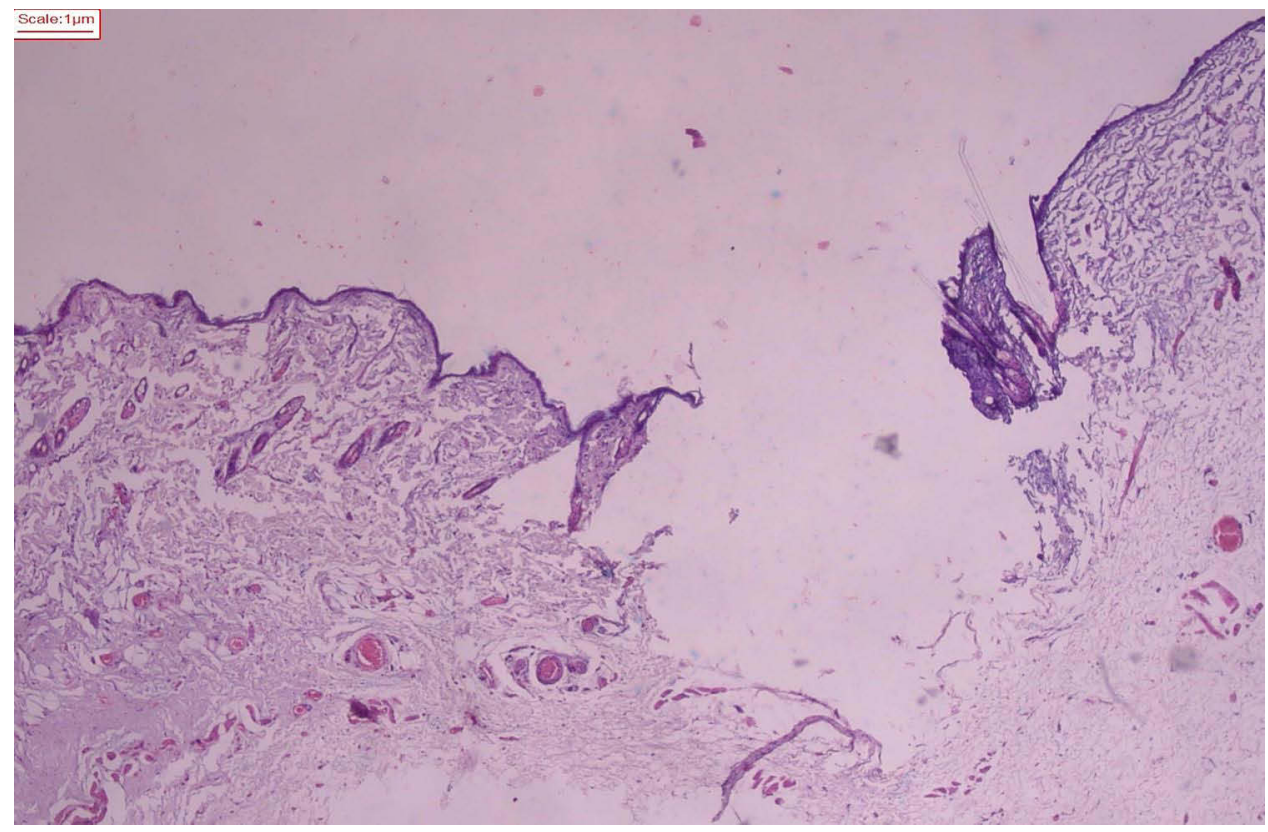

Figure I Histopathological view of the deep dermal burn model with hematoxylin-eosin staining shows the absence of the epidermal and dermal layers of the skin (HE staining, magnification $40 x$ ).

0.050), so it can be concluded that there was an insignificant difference among interventions (Figure 3).

\section{Tissue Malondialdehyde Level at 48 Hours}

Based on Table 1, the highest average 48-hour tissue MDA rate was in the negative control group $(1.523 \mu \mathrm{mol} / \mathrm{L} \pm 0.256)$, and the lowest was in the treatment group $(0.570 \mu \mathrm{mol} / \mathrm{L} \pm 0.656)$.
The Kruskal-Wallis test showed that the p-value was smaller than $\alpha(0.041<0.050)$, so it can be concluded that there was a significant difference. To see where the difference was, the Mann-Whitney test was performed, with the following results: the treatment group differed significantly from the positive control group and negative control group, with a significance value of 0.050 . The treatment group was not substantially different from the normal

Table I Measurement Results for Malondialdehyde and Nitric Oxide Levels and p-values (One-Way ANOVA)

\begin{tabular}{|l|l|l|l|l|l|}
\hline Time Points & Group of Intervention & Blood MDA Level & Tissue MDA Level & Blood NO Level & Tissue NO Level \\
\hline 8th hours & Treatment Group & $0.550( \pm 0.113)$ & $0.593( \pm 0.050)$ & $15.333( \pm 3.055)$ & $20.667( \pm 1.528)$ \\
& Positive Control & $0.603( \pm 0.065)$ & $0.687( \pm 0.029)$ & $40.333( \pm 9.866)$ & $35.000( \pm 3.605)$ \\
& Negative Control & $0.813( \pm 0.078)$ & $0.843( \pm 0.125)$ & $48.333( \pm 4.619)$ & $33.667( \pm 2.309)$ \\
& Normal Control & $0.553( \pm 0.067)$ & $0.657( \pm 0.100)$ & $14.000( \pm 1.000)$ & $18.333( \pm 1.528)$ \\
& & $\mathrm{P}=0.014^{*}$ & $\mathrm{P}=0.036^{*}$ & $\mathrm{p}=0.000^{*}$ & $\mathrm{P}=0.000^{*}$ \\
\hline \multirow{2}{*}{ 44th hours } & Treatment Group & $0.537( \pm 0.185)$ & $0.677( \pm 0.076)$ & $19.333( \pm 4.509)$ & $18.000( \pm 7.21 \mathrm{I})$ \\
& Positive Control & $0.703( \pm 0.015)$ & $0.730( \pm 0.061)$ & $28.333( \pm 4.619)$ & $16.667( \pm 6.028)$ \\
& Negative Control & $0.820( \pm 0.171)$ & $0.767( \pm 0.081)$ & $36.000( \pm 1.000)$ & $28.000( \pm 2.646)$ \\
& Normal Control & $0.547( \pm 0.064)$ & $0.650( \pm 0.096)$ & $13.333( \pm 0.577)$ & $18.667( \pm 1.528)$ \\
& & $\mathrm{P}=0.080$ & $\mathrm{P}=0.339$ & $\mathrm{P}=0.000^{*}$ & $\mathrm{P}=0.079$ \\
\hline \multirow{2}{*}{48 th hours } & Treatment Group & $0.593( \pm 0.137)$ & $0.570( \pm 0.656)$ & $21.667( \pm 3.786)$ & $3.333( \pm 3.519)$ \\
& Positive Control & $0.880( \pm 0.072)$ & $0.713( \pm 0.032)$ & $24.667( \pm 2.517)$ & $31.667( \pm 9.713)$ \\
& Negative Control & $0.813( \pm 0.035)$ & $1.523( \pm 0.256)$ & $37.333( \pm 5.859)$ & $51.667( \pm 6.110)$ \\
& Normal Control & $0.550( \pm 0.069)$ & $0.653( \pm 0.104)$ & $15.000( \pm 1.000)$ & $15.667( \pm 1.155)$ \\
& & $\mathrm{P}=0.004^{*}$ & $\mathrm{P}=0.04 I^{*}$ & $\mathrm{P}=0.00 \mathrm{I}^{*}$ & $\mathrm{P}=0.000^{*}$ \\
\hline
\end{tabular}

Note: $* 95 \%$ confidence interval. 


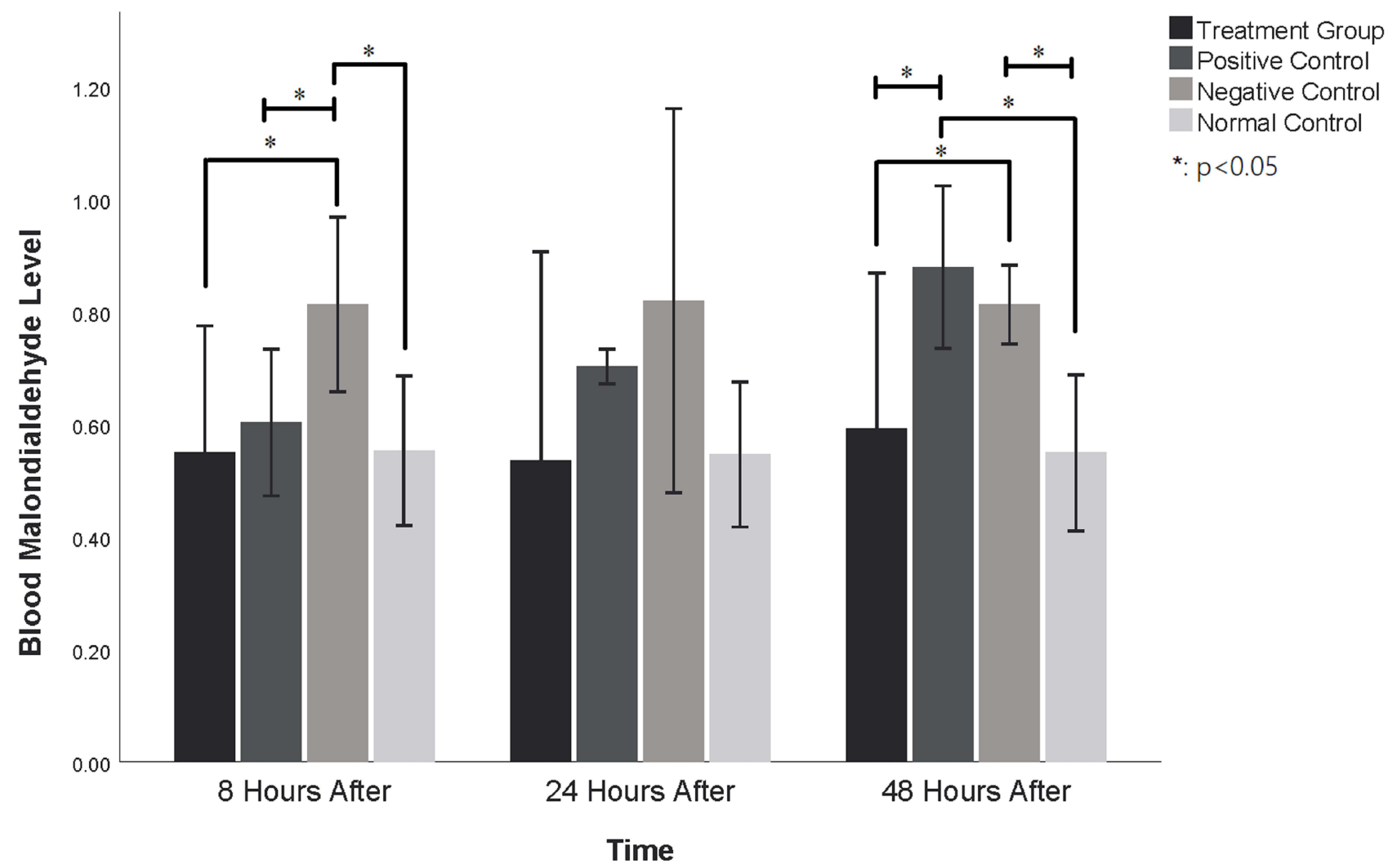

Figure 2 Multiple comparisons of blood malondialdehyde levels. ${ }^{*} p<0.05$.

control group, with a value of $p=0.376$. The positive control group differed significantly from the treatment group and negative control group but was not significantly different from the normal control group, with a significance value of 0.513 (Figure 3).

\section{Blood Nitric Oxide Level at 8 Hours}

Based on Table 1, the highest average blood NO level at 8 hours was in the negative control group $(48.333 \mathrm{nmol} / \mathrm{mg}$ \pm 4.619 ), and the lowest average value was in the normal control group, followed by the treatment group (14.000 $\mathrm{nmol} / \mathrm{mg} \pm 1.000$ and $15.333 \mathrm{nmol} / \mathrm{mg} \pm 3.055$, respectively).

The NO blood level at 8 hours showed a significantly different result, with $p=0.000$ (Table 1 ). Then, to determine the value of the differences among groups, multiple comparisons were performed with the LSD test (Figure 4). The combination of SVFs and PRP showed a statistically significant difference compared to the Vaseline group $(\mathrm{p}=0.0007)$ or negative control group $(p=0.0001)$. There was no significant difference compared to the normal control group.

The positive control group showed a significant difference from the treatment group $(p=0.0001)$ and normal control group $(p=0.0006)$. However, there was no significant difference from the negative control group. The negative control group showed a statistically significant difference compared to the treatment group $(p=0.0001)$ and normal control group $(\mathrm{p}=0.0001)$.

\section{Blood Nitric Oxide Level at 24 Hours}

Based on Table 1, the highest average 24-hour blood NO level was in the negative control group $(36.000 \mathrm{nmol} / \mathrm{mg} \pm$ $1.000)$, and the lowest value was in the normal control group, followed by the treatment group $(13.333 \mathrm{nmol} / \mathrm{mg}$ \pm 0.577 and $19.333 \mathrm{nmol} / \mathrm{mg} \pm 4.509$, respectively). Oneway ANOVA showed a significant result, with $\mathrm{p}=0.000$ (Table 1). The results of the LSD test (Figure 4) showed that the treatment group was significantly different from the positive control group $(p=0.009)$ and the negative control group $(p=0.0003)$. There was no difference compared to the normal control group.

The positive control group showed a significant difference compared to the treatment group $(\mathrm{p}=0.0099)$, negative control group (0.021) and normal control group $(\mathrm{p}=$ 0.0005). The negative control group showed a significant difference compared to the treatment group $(\mathrm{p}=0.0003)$, 


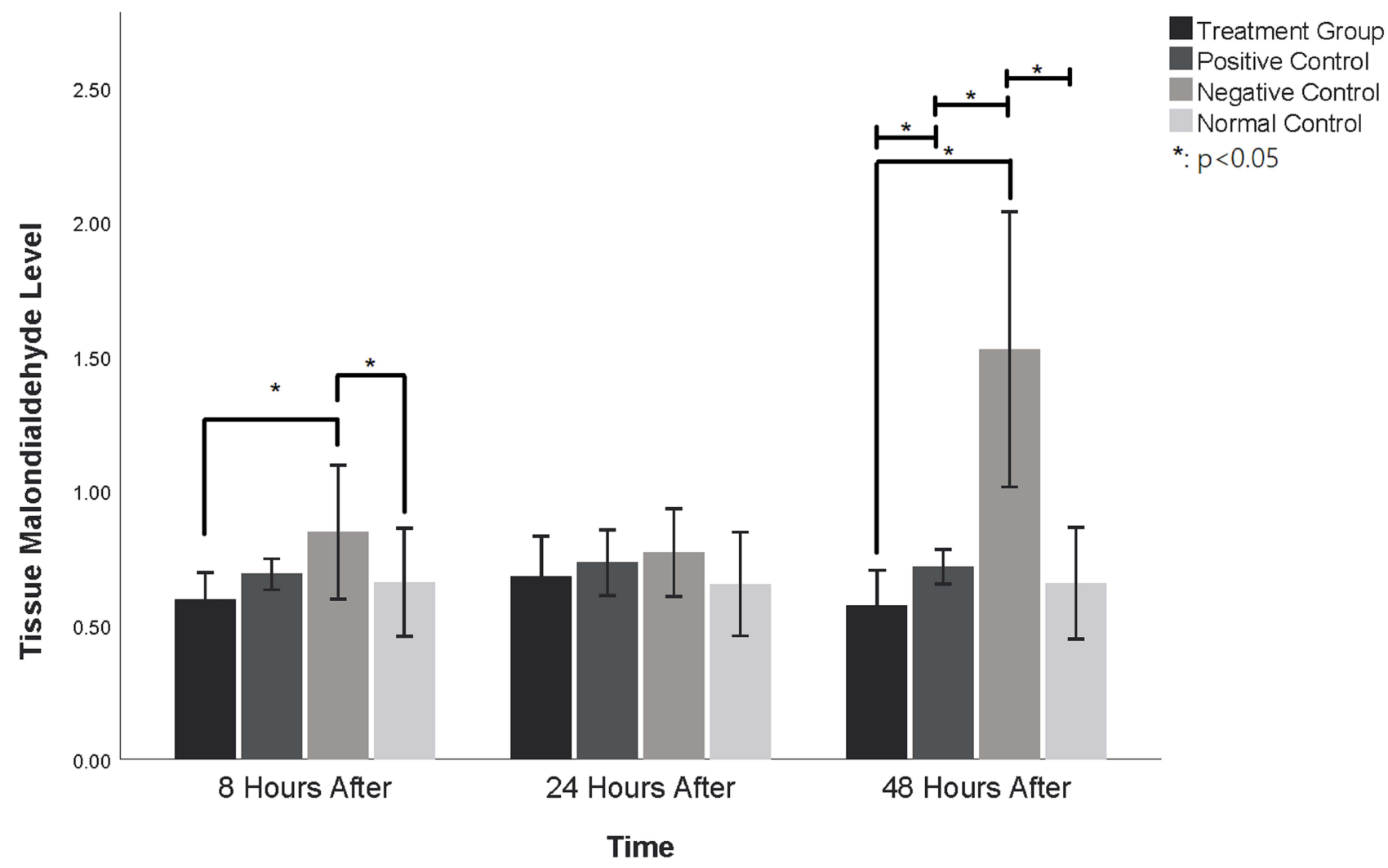

Figure 3 Multiple comparisons of tissue malondialdehyde levels. ${ }^{*} \mathrm{p}<0.05$.

positive control group $(\mathrm{p}=0.021)$ and normal control group $(\mathrm{p}=0.0001)$ (Figure 4$)$.

\section{Blood Nitric Oxide Level at 48 Hours}

Based on Table 1, the highest average blood NO level at 48 hours was in the negative control group (37.333 $\mathrm{nmol} / \mathrm{mg} \pm 5.859$ ), and the lowest value was in the normal control group, followed by the treatment group $(15.000 \mathrm{nmol} / \mathrm{mg} \pm 1.000$ and $21.667 \mathrm{nmol} / \mathrm{mg} \pm 3.786$, respectively). One-way ANOVA showed a p-value of 0.001 , indicating that there were differences among interventions.

The LSD test showed that the treatment group was significantly different from the negative control group $(\mathrm{p}$ $=0.001)$ but not significantly different from the positive control group or normal control group $(\mathrm{p}=0.355$ and $\mathrm{p}=$ 0.61 , respectively). The negative control group showed a significant difference compared to the treatment group $(p=0.001)$, positive control group $(p=0.003)$ and normal control group $(p=0.000)$. The normal control group showed no significant difference compared to the treatment group $(p=0.06)$ (Figure 4).

\section{Tissue Nitric Oxide Level at 8 Hours}

Based on Table 1, the highest tissue NO level at 8 hours was in the positive control group, followed by the negative control group $(35.000 \mathrm{nmol} / \mathrm{mg} \pm 3.605$ and $33.667 \mathrm{nmol} / \mathrm{mg} \pm 2.309$, respectively). The lowest tissue NO level was in the normal control group, followed by the treatment group $(18.333 \mathrm{nmol} /$ $\mathrm{mg} \pm 1.53$ and $20.667 \mathrm{nmol} / \mathrm{mg} \pm 1.528$, respectively).

One-way ANOVA showed a significant difference among interventions, with $p=0.000$. The LSD test showed that the treatment group was significantly different from the positive control group and the negative control group $(p=0.000$ and $\mathrm{p}=0.000$, respectively). However, it was insignificantly different from the normal control group $(p=0.268)$ (Figure 5).

\section{Tissue Nitric Oxide Level at 24 Hours}

Based on Table 1, the highest average 24-hour tissue NO level was in the negative control group $(28.000 \mathrm{nmol} / \mathrm{mg} \pm$ $2.646)$, and the lowest value was in the positive control group, followed by the treatment group $(16.667 \mathrm{nmol} / \mathrm{mg}$ \pm 6.028 and $18.000 \mathrm{nmol} / \mathrm{mg} \pm 7.211$, respectively). Oneway ANOVA showed that the results were not significantly different $(p=0.079)$ (Figure 5). 


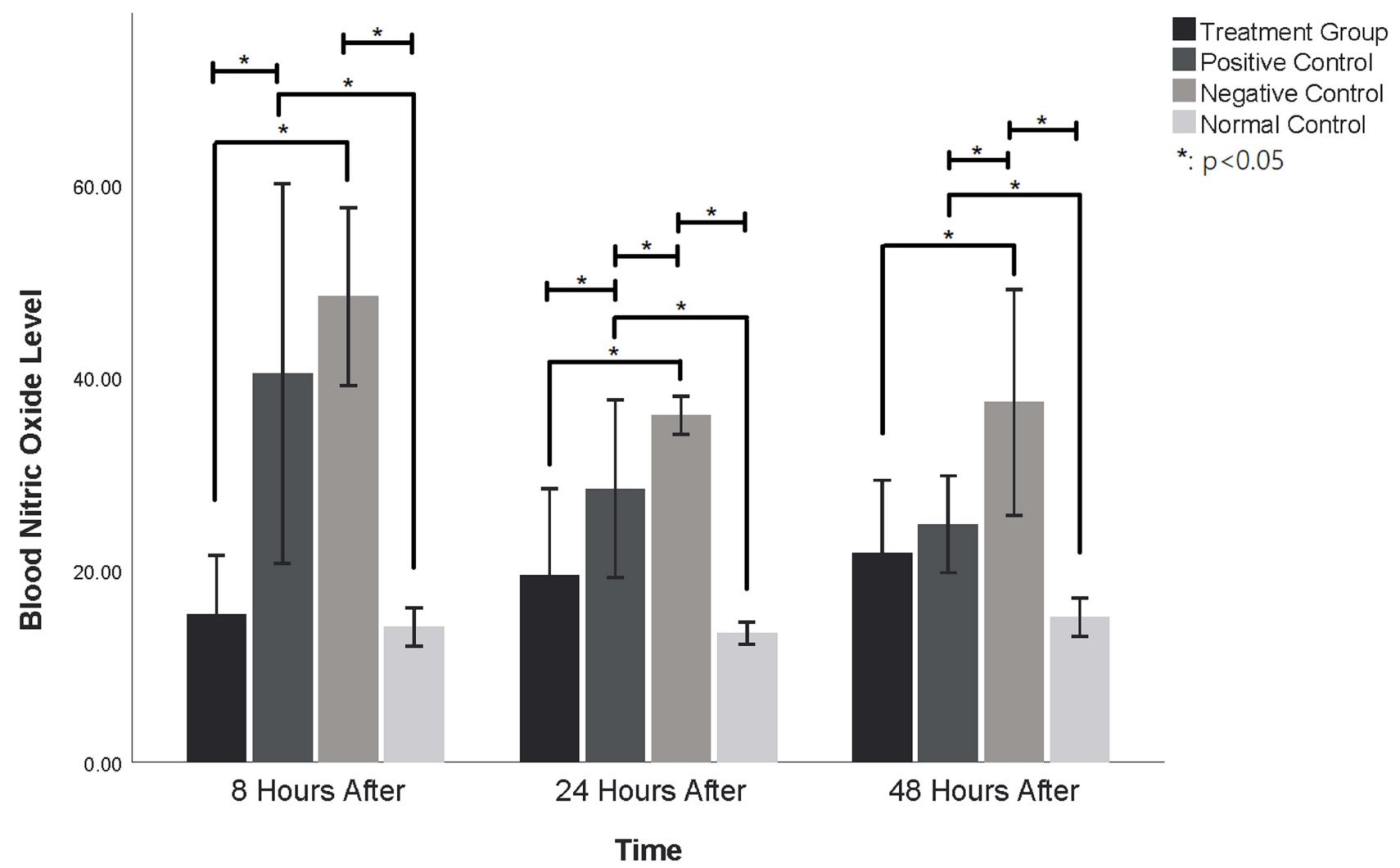

Figure 4 Multiple comparisons of blood nitric oxide levels. ${ }^{*} p<0.05$.

\section{Tissue Nitric Oxide Level at 48 Hours}

Based on Table 1, the highest average tissue NO level was in the negative control group $(51.667 \mathrm{nmol} / \mathrm{mg} \pm 6.110)$, and the lowest value was in treatment group, followed by the normal control group $(3.333 \mathrm{nmol} / \mathrm{mg} \pm 3.519$ and $15.667 \mathrm{nmol} / \mathrm{mg} \pm 1.155$, respectively). One-way ANOVA showed significantly different results $(\mathrm{p}=$ 0.000). The LSD test showed that the treatment group was significantly different from the positive control group, negative control group, and normal control group $(p=0.000, p=0.000$ and $p=0.037$, respectively) (Figure 5).

\section{Regression Test Results}

A regression test was conducted to evaluate the effect of locally injected SVFs and PRP in combination. It was also conducted for the positive control group to distinguish which group had reduced MDA and NO levels.

As showed in Table 2, local injection of SVFs and PRP in combination had an effect on blood and tissue MDA and NO levels $(\mathrm{P}<0.05)$. A regression test for the treatment group was carried out with the following results:
- Regarding the blood MDA level, the R-square value of $0.766(76.6 \%)$ indicated that local injection of SVFs and PRP in combination reduced blood MDA levels by $76.6 \%$, while the remaining levels $(23.4 \%)$ were influenced by other factors. The equation ( $\mathrm{Y}=$ $0.817+(-0.257 \mathrm{X}))$ indicates that each injection of SVFs and PRP in combination will decrease the blood MDA level by $0.257 \mu \mathrm{mol} / \mathrm{L}$.

- Regarding the tissue MDA level, the R-square value of 0.932 (93.2\%) indicated that the local injection of SVFs and PRP in combination reduced MDA tissue levels by $93.2 \%$, while the remaining levels $(6.8 \%)$ were influenced by other factors. The equation $(\mathrm{Y}=$ $1.043+(-0.427 \mathrm{X})$ indicates that each injection of SVFs and PRP in combination will decrease the tissue MDA level by $0.427 \mu \mathrm{mol} / \mathrm{L}$.

- Regarding the blood NO level, the R-square value of $0.968(96.8 \%)$ indicated that local injection of SVFs and PRP in combination reduced NO blood levels by $96.8 \%$, while the remaining levels $(3.2 \%)$ were influenced by other factors. The equation $(\mathrm{Y}=40.557+(-21.78 \mathrm{X}))$ indicates that each injection of SVFs and PRP in 


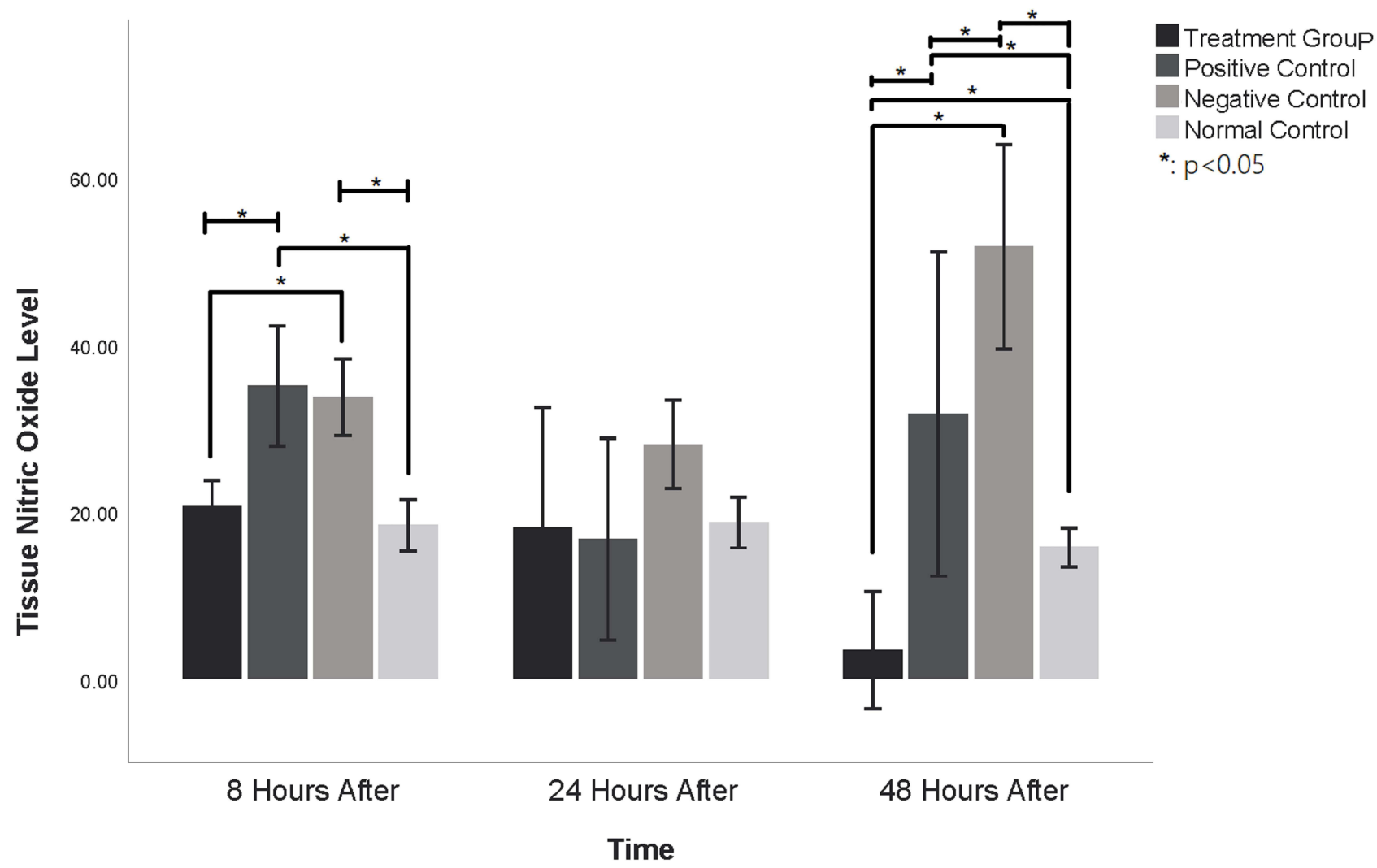

Figure 5 Multiple comparisons of tissue nitric oxide levels. ${ }^{*} p<0.05$.

combination will decrease the NO blood level by 21.78 $\mathrm{nmol} / \mathrm{mg}$.

- Regarding the tissue NO level, R-square was 0.982 (98.2\%), which indicated that the local injection of SVFs and PRP in combination reduced NO tissue levels by $98.2 \%$, while the remaining levels $(1.8 \%)$ were influenced by other factors. The equation ( $\mathrm{Y}=$ $37.778+(-23.777 \mathrm{X}))$ indicates that each injection of SVFs and PRP in combination will decrease the NO tissue level by $23.777 \mathrm{nmol} / \mathrm{mg}$.

As shown in Table 3, Vaseline had an effect on tissue MDA levels, blood NO levels $(\mathrm{P}<0.05)$, and tissue NO levels while the rest of the experiments showed insignificant results. A regression test for the positive control group was carried out with the following results:

- Regarding the blood MDA level, the P-value of 0.114 $(>0.05)$ indicated that there was no significant effect of topically applied Vaseline on MDA blood levels.

- Regarding the tissue MDA level, the R-square value of $0.898(89.8 \%)$ indicated that topical application of Vaseline influenced MDA tissue levels by $89.8 \%$, while the remaining levels (10.2\%) were influenced by other factors. The equation $(\mathrm{Y}=1.043+$ $(-0.333 \mathrm{X}))$ indicated that each Vaseline application would decrease the tissue MDA level by $0.333 \mu \mathrm{mol} / \mathrm{L}$.

Table 2 Regression Test Results in the Treatment Group

\begin{tabular}{|l|l|l|l|l|}
\hline & P-value & $\mathbf{R}$ & R-Square & Equation \\
\hline MDA Blood & $0.022^{*}$ & 0.875 & 0.766 & $Y=0.817+(-0.257 X)$ \\
MDA Tissue & $0.002^{*}$ & 0.965 & 0.932 & $Y=1.043+(-0.427 X)$ \\
NO Blood & $0.000^{*}$ & 0.984 & 0.968 & $Y=40.557+(-21.78 X)$ \\
NO Tissue & $0.000^{*}$ & 0.991 & 0.982 & $Y=37.777+(-23.777 X)$ \\
\hline
\end{tabular}

Note: $* 95 \%$ confidence interval. 
Table 3 Regression Test Results in the Positive Control Group

\begin{tabular}{|l|l|l|l|l|}
\hline & P-value & R & R-Square & Equation \\
\hline MDA Blood & 0.114 & 0.710 & 0.504 & $Y=0.817+(-0.090 X)$ \\
MDA Tissue & $0.004^{*}$ & 0.948 & 0.898 & $Y=1.043+(-0.333 X)$ \\
NO Blood & $0.028^{*}$ & 0.861 & 0.742 & $Y=40.557+(-9.447 X)$ \\
NO Tissue & $0.04 I^{*}$ & 0.830 & 0.689 & $Y=37.777+(-10 X)$ \\
\hline
\end{tabular}

Note: $* 95 \%$ confidence interval.

- Regarding the blood NO level, the R-square value of $0.742(74.2 \%)$ indicated that Vaseline reduced NO blood levels by $74.2 \%$, while the remaining levels $(25.8 \%)$ were influenced by other factors. The equation $(\mathrm{Y}=40.557+(-9.447 \mathrm{X}))$ indicated that each Vaseline application would decrease the blood NO level by $9.447 \mathrm{nmol} / \mathrm{mg}$.

- Regarding the tissue NO level the R-square value of $0.689(68.9 \%)$ indicated that Vaseline reduced NO tissue levels by $68.9 \%$, while the remaining levels (31.1\%) were influenced by other factors. The equation $(\mathrm{Y}=$ $37.777+(-10 \mathrm{X}))$ indicated that each Vaseline application would decrease the tissue $\mathrm{NO}$ level by $10 \mathrm{nmol} / \mathrm{mg}$.

In summary regression test result the treatment showed a greater reduction in MDA and NO levels than the positive control group, with the following results:

- Regarding blood MDA levels, injection of SVFs and PRP in combination reduced MDA blood levels by $0.257 \mu \mathrm{mol} / \mathrm{L}$, while in the positive control group, application of Vaseline did not affect blood MDA levels.

- Regarding tissue MDA levels, injection of SVFs and PRP in combination reduced tissue MDA levels by 1.282 times compared with Vaseline application.

- Regarding blood NO levels, injection of SVFs and PRP in combination reduced blood NO levels by 2.305 times compared with Vaseline application.

- Regarding tissue NO levels, injection of SVFs and PRP in combination reduced tissue NO levels by 2.378 times, while in the positive control group, application of Vaseline did not affect tissue NO levels.

\section{Discussion}

Burn injury causes local and systemic responses in the body. Locally, tissue ischemia and inflammation will lead to necrosis if not treated in a prompt manner; a zone of stasis will become a zone of necrosis. In extensive burn injury, defined as $20 \%$ or more of total body surface area (TBSA) affected or $10 \%$ of TBSA affected for children, there are systemic consequences that result in the release of pro-inflammatory mediators and the production of ROS and RNS. ${ }^{2,3}$

Activated neutrophils and macrophages are one mechanism by which ROS are formed. These leucocytes require ROS as free radicals to form oxidants that act as physiological antimicrobials. In addition, the physiological process of ROS formation can modulate lymphocytes, inducing them to become more potent immune agents. However, when the homeostasis of these oxidants is disturbed, there is an overload of oxidants and destructive oxidative stress. ${ }^{23-28}$ In burn patients, neutrophils accumulate in tissues such as the gastric mucosa, liver and lungs, which will then be followed by the formation of ROS, explaining why organ damage occurs in a different place from the primary location of the burn. ${ }^{29}$

Another mechanism by which ROS form is fatty acid peroxidation, which is a reaction of oxygen with unsaturated lipids. One of the pathways for lipid peroxidation involves the biosynthesis of thromboxane A2 (TXA2). TXA2 itself is an active metabolite of the cyclooxygenase process that is involved in arachidonic acid production. The second route is the reaction with oxygen radicals. In the end, these pathways form secondary products, namely, aldehydes, one of which is MDA. In contrast to free radicals in general, these products of lipid peroxidation easily diffuse and circulate throughout tissues that are far from the primary location. ${ }^{7}$ This could be the reason why a burn injury that is initially localized (increased tissue MDA levels) can become a systemic problem; in this study, this was illustrated by the increased blood MDA levels.

NO is a direct product of activated macrophages due to oxidative stress in burns. When there is severe inflammation in the tissue, there will be a burst of NO production through the inducible Nos synthesis (iNOS) mechanism. When the NO level exceeds a certain limit 
and exceeds other protective cofactors, NO will react with oxygen radicals to form RNS. RNS can oxidize, titrate, decompose, and produce more destructive species. NO is an important mediator of immunosuppressants after burn injury and is mainly responsible for inhibiting the proliferative response through the formation of peroxynitrite and its acid conjugate. This will eventually result in damage to genomic DNA, with the depletion of cell energy and cell death. ${ }^{29}$ In other words, in this study, ROS were measured through the assessment of the metabolite MDA, and RNS was measured through the assessment of the mediator NO.

MDA and NO levels will increase in the early phase of burn injury and decrease over time. In this study, MDA and NO levels were significantly increased $(\mathrm{P}<0.050)$ compared to those in the normal control group (Figures 2-5). This indicates that when burns occur, inflammatory and oxidative reactions follow both local and systemic processes.

SVFs are known to act as agents that accelerate the healing process of burn wounds, with fibroplasia, angiogenesis, modulation of inflammatory cells to become constructive cells and increased collagen synthesis. Similarly, PRP is very rich in growth factors that play a role in wound healing by inducing inflammatory cell migration, cell proliferation and cell differentiation. In summary, the potential antioxidant and anti-inflammatory effects of these two agents make the combination of SVFs and PRP a very promising, new and interesting therapeutic approach for burn patients. ${ }^{30,31}$

This study showed that MDA and NO levels in the treatment group were lower than those in the negative control group (placebo). This indicates that the injection of both SVFs and PRP significantly reduced oxidative stress. As reported by Kim et al, ${ }^{32}$ SVFs contain IGF, pigment epithelium-derived factor (PEDF), secreted superoxide dismutase, and glutathione peroxidase, and PRP contains many growth factors, such as IGF-1 and PDGF, as protective agents against free radical toxins. ${ }^{14}$

In this study, it was found that the blood MDA levels in the treatment group (combination of SVFs and PRP) were significantly different from those in the positive control group (Vaseline) at 48 hours but not at 8 and 24 hours prior to burn injury (Figure 2). This may have occurred because Vaseline has natural antioxidant and local antiinflammatory effects. ${ }^{33}$ In addition, MDA is a byproduct of lipid peroxidation and parameters of severe tissue necrosis that may not occur 6 hours after burn injury, but due to the prolonged inflammatory response and the overproduction of MDA over time, Vaseline is not able to maintain its antioxidant and anti-inflammatory activities.

Tissue NO levels in the treatment group (combination of SVFs and PRP) showed statistically significant results compared to the positive control group (Vaseline) at 8 and 48 hours after injection. However, blood NO levels showed insignificant results at 48 hours. This may be because NO is a direct product of activated macrophages due to oxidative stress, which is affected locally by burn injury. Local injection of SVFs and PRP has a local effect by reducing tissue NO production. Local injection of the combination of SVFs and PRP may also suppress the production of ROS and RNS and balance the production of inflammatory and anti-inflammatory mediators so that oxidative stress can be properly maintained.

Local injection of SVFs and PRP induces the healing phase earlier, suppresses the production of ROS/RNS, and prevents the burn zone from becoming deeper. This was proven to be statistically significant in this study; in the treatment group, blood and tissue MDA and NO levels decreased and are approaching with those in the normal control group. The combination treatment also shows better long-term effects in the later phase of burn injury. The more severe the tissue damage is, the longer the inflammatory response. Similarly, the longer the healing process starts, the more toxic compounds are excreted. 4,34

This study found that the local injection of SVFs and PRP in combination decreased blood MDA, tissue MDA, blood NO and tissue NO levels by $76.6 \%, 93.2 \%, 96.8 \%$, and $98.2 \%$, respectively, while the remaining MDA and NO levels were influenced by other factors. This study also found that local injection of SVFs and PRP in combination reduced MDA and NO levels better than Vaseline, a reduction of 1.282-fold was observed for tissue MDA levels, a reduction of 2.305-fold was observed for blood NO levels and a reduction of 2.378-fold was observed for tissue $\mathrm{NO}$ levels. In particular, Vaseline showed no significant effects on blood MDA; while the SVFs and PRP in combination treatment reduced blood MDA levels by $0.257 \mu \mathrm{mol} / \mathrm{L}$ after injection. This could occur because MDA was constantly formed in areas distant from the primary site of the injury, while Vaseline mostly acted as a local antioxidant. NO levels in tissue were higher at 48 hours than at 24 hours, which suggests that NO levels began to rise as burn injury continued to develop. This shared the same trend as NO blood levels but not as high as NO tissue levels. NO was primarily formed in tissue and eventually released into the blood circulation, 
which could be the reason why the NO blood level 48 hours after injury was lower than the NO tissue level 48 hours after injury. This could be the reason why Vaseline reduced NO levels in the tissue but not the blood.

The effectiveness of the combination SVFs and PRP against burns of various depths also needs to be studied further, especially in full-thickness burn injuries. In daily medical practice, the treatment of early-phase burn injury is a critical stage in determining the future prognosis of patients. Although it was proven in this study that the combination of SVFs and PRP reduced MDA and NO levels, emergency treatment for severe-critical burn injury requires much time and attention from medical personnel. In addition, the preparation of SVFs and PRP is relatively difficult to perform in an emergency setting in critical burn patients. This highlights the usage of autologous SVFs combination PRP in emergency room remains a challenge.

\section{Conclusion}

Local injection of SVFs and PRP in combination is a new idea and a breakthrough in burn management. In this study, it was proven that the combination of these two preparations undeniably reduced the MDA and NO levels in blood and tissue compared to those of the Vaseline and placebo groups. This result indicates that the combined injection of these two preparations inhibits the local response to burn injury, which prevents the occurrence of a severe systemic inflammatory burn response.

\section{Author Contributions}

All authors made a significant contribution to the work reported, whether that is in the conception, study design, execution, acquisition of data, analysis and interpretation, or in all these areas; took part in drafting, revising or critically reviewing the article; gave final approval of the version to be published; have agreed on the journal to which the article has been submitted; and agree to be accountable for all aspects of the work.

\section{Disclosure}

The authors have no financial or other conflicts of interest regarding the publication of this article.

\section{References}

1. Kaddoura I, Abu-Sittah G, Ibrahim A, Karamanoukian R, Papazian N. Burn injury: review of pathophysiology and therapeutic modalities in major burns. Ann Burns Fire Disasters. 2017;30(2):95-102.
2. Stander M, Wallis LA. The emergency management and treatment of severe burns. Emerg Med Int. 2011;2011:161375. doi:10.1155/2011/ 161375

3. Strudwick XL, Cowin AJ. The role of the inflammatory response in burn injury. In: Hot Topics in Burn Injuries. InTech; 2018:38-61. doi:10.5772/intechopen.71330

4. Salibian AA, Rosario ATD, Severo LDAM, et al. Current concepts on burn wound conversion-A review of recent advances in understanding the secondary progressions of burns. Burns. 2016;42 (5):1025-1035. doi:10.1016/j.burns.2015.11.007

5. Babu R, Babu M. Oxidative stress in major thermal burns: its implications and significance. Indian J Burn. 2018;26(1):38. doi:10.4103/ ijb.ijb_37_17

6. Aydemir S, Akgün SG, Beceren A, et al. Melatonin ameliorates oxidative DNA damage and protects against formaldehyde-induced oxidative stress in rats. 2017.

7. Ayala A, Muñoz MF, Argüelles S. Lipid peroxidation: production, metabolism, and signaling mechanisms of malondialdehyde and 4-hydroxy-2-nonenal. Oxid Med Cell Longev. 2014;2014:360438. doi: $10.1155 / 2014 / 360438$

8. Rawlingson A. Nitric oxide, inflammation and acute burn injury. Burns. 2003;29(7):631-640. doi:10.1016/S0305-4179(03)00079-2

9. Szabó C, Ischiropoulos H, Radi R. Peroxynitrite: biochemistry, pathophysiology and development of therapeutics. Nat Rev Drug Discov. 2007;6(8):662-680. doi:10.1038/nrd2222

10. Laidding SR, Josh F, Faruk M, et al. Combination of platelet-rich plasma and stromal vascular fraction on the level of transforming growth factor- $\beta$ in rat subjects experiencing deep dermal burn injury. Ann Med Surg. 2020;60:737-742. doi:10.1016/j. amsu.2020.11.088

11. Josh F, Tobita M, Tanaka R, et al. Concentration of PDGF-AB, BB and TGF- $\beta 1$ as valuable human serum parameters in adipose-derived stem cell proliferation. J Nippon Med Sch. 2013;80(2):140-147. doi: $10.1272 /$ jnms. 80.140

12. Ramakrishnan VM, Boyd NL. The adipose stromal vascular fraction as a complex cellular source for tissue engineering applications. Tissue Eng Part B Rev. 2018;24(4):289-299. doi:10.1089/ten. teb.2017.0061

13. Nava S, Sordi V, Pascucci L, et al. Long-lasting anti-inflammatory activity of human microfragmented adipose tissue. Stem Cells Int. 2019;2019:1-13. doi:10.1155/2019/5901479

14. Unal M. Platelet-rich plasma in burn treatment. In Hot Topics in Burn Injuries. InTech; 2018. doi10.5772/intechopen.70835

15. Mansoub NH, Gürdal M, Karadadas E, Kabadayi H, Vatansever S, Ercan G. The role of PRP and adipose tissue-derived keratinocytes on burn wound healing in diabetic rats. BioImpacts. 2018;8(1):5-12. doi:10.15171/bi.2018.02

16. Laidding SR, Josh F, Battung S, et al. Combination of platelet rich plasma and stromal vascular fraction on the level of vascular endothelial growth factor in rat subjects experiencing deep dermal burn injury. Ann Med Surg. 2021;64:102254. doi:10.1016/j. amsu.2021.102254

17. Laidding SR, Josh F, Nur K, et al. The effect of combined platelet-rich plasma and stromal vascular fraction compared with platelet-rich plasma, stromal vascular fraction, and vaseline alone on healing of deep dermal burn wound injuries in the Wistar rat. Med Clínica Práctica. 2021;4:100239. doi:10.1016/j. mcpsp.2021.100239

18. Chieregato K, Castegnaro S, Madeo D, Astori G, Pegoraro M, Rodeghiero F. Epidermal growth factor, basic fibroblast growth factor and platelet-derived growth factor-bb can substitute for fetal bovine serum and compete with human platelet-rich plasma in the ex vivo expansion of mesenchymal stromal cells derived from adipose tiss. Cytotherapy. 2011;13(8):933-943. doi:10.3109/14653249.2011. 583232 
19. Kilkenny C, Browne WJ, Cuthill IC, Emerson M, Altman DG. Improving bioscience research reporting: the ARRIVE guidelines for reporting animal research. PLoS Biol. 2010;8(6):e1000412. doi:10.1371/journal.pbio. 1000412

20. Tobita M, Tajima S, Orbay H, Hyakusoku H, Mizuno H. Bone regeneration by the combined implantation of adipose-derived stem cells and platelet-rich plasma. Wound Repair Regen. 2014;22:A18.

21. Josh F, Kobe K, Tobita M, et al. Accelerated and safe proliferation of human adipose-derived stem cells in medium supplemented with human serum. J Nippon Med Sch. 2012;79(6):444-452. doi:10.1272/jnms.79.444

22. Tavares Pereira DD, Lima-Ribeiro MH, de Pontes-filho NT, et al. Development of animal model for studying deep second-degree thermal burns. J Biomed Biotechnol. 2012;2012:460841. doi:10.1155/ $2012 / 460841$

23. Hampton MB, Kettle AJ, Winterbourn CC. Inside the neutrophil phagosome: oxidants, myeloperoxidase, and bacterial killing. Blood. 1998;92(9):3007-3017. doi:10.1182/blood.V92.9.3007

24. Schoonbroodt S, Legrand-Poels S, Best-Belpomme M, Piette J. Activation of the NF- $\mathrm{B}$ transcription factor in a T-lymphocytic cell line by hypochlorous acid. Biochem J. 1997;321(3):777-785. doi:10.1042/bj3210777

25. Schoonbroodt S, Piette J. Oxidative stress interference with the nuclear factor- $\kappa \mathrm{B}$ activation pathways. Biochem Pharmacol. 2000;60(8):1075-1083. doi:10.1016/S0006-2952(00)00371-3

26. Steinbeck M, Khan AU, Karnovsky M. Extracellular production of singlet oxygen by stimulated macrophages quantified using 9,10-diphenylanthracene and perylene in a polystyrene film. $J$ Biol Chem. 1993;268:15649-15654. doi:10.1016/S0021-9258(18)82305-5
27. Bonizzi G, Piette J, Merville MP, Bours V. Cell type-specific role for reactive oxygen species in nuclear factor-kappaB activation by Interleukin-1. Biochem Pharmacol. 2000;59(1):7-11. doi:10.1016/ S0006-2952(99)00290-7

28. Fahn S, Cohen G. The oxidant stress hypothesis in Parkinson's disease: evidence supporting it. Ann Neurol. 1992;32(6):804-812. doi:10.1002/ana.410320616

29. Parihar A, Parihar MS, Milner S, Bhat S. Oxidative stress and anti-oxidative mobilization in burn injury. Burns. 2008;34(1):6-17. doi:10.1016/j.burns.2007.04.009

30. Yan H, Xing X, Li H, Yan H, Li X, Zhu J. Role of stromal vascular fraction in severe burn wound healing process. Biomed Res. 2017;28 (3).

31. Cardoso AL, Bachion MM, Morais JD, et al. Adipose tissue stromal vascular fraction in the treatment of full thickness burns in rats. Acta Cir Bras. 2016;31(9):578-585. doi:10.1590/S0102-86502016009 0000002

32. Kim WS, Park BS, Sung JH. The wound-healing and antioxidant effects of adipose-derived stem cells. Expert Opin Biol Ther. 2009;9 (7):879-887. doi:10.1517/14712590903039684

33. Sethi A, Kaur T, Malhotra SK, Gambhir ML. Moisturizers: the slippery road. Indian J Dermatol. 2016;61(3):279-287. doi:10.4103/ 0019-5154.182427

34. Singer AJ, Toussaint J, Chung WT, McClain S, Raut V, Rosenberg L. The effects of platelet rich plasma on healing of full thickness burns in swine. Burns. 2018;44(6):1543-1550. doi:10.1016/j. burns.2018.04.021
Journal of Inflammation Research

\section{Publish your work in this journal}

The Journal of Inflammation Research is an international, peerreviewed open-access journal that welcomes laboratory and clinical findings on the molecular basis, cell biology and pharmacology of inflammation including original research, reviews, symposium reports, hypothesis formation and commentaries on: acute/chronic inflammation; mediators of inflammation; cellular processes; molecular mechanisms; pharmacology and novel anti-inflammatory drugs; clinical conditions involving inflammation. The manuscript management system is completely online and includes a very quick and fair peerreview system. Visit http://www.dovepress.com/testimonials.php to read real quotes from published authors. 